\title{
Adaptive Power Allocation and Splitting with Imperfect Channel Estimation in Energy Harvesting Based Self-Organizing Networks
}

\author{
Kisong Lee ${ }^{1}$ and JeongGil Ko ${ }^{2}$ \\ ${ }^{1}$ Department of Information and Telecommunication Engineering, Kunsan National University, Gunsan 573-701, Republic of Korea \\ ${ }^{2}$ Department of Software and Computer Engineering, Ajou University, Suwon 16499, Republic of Korea \\ Correspondence should be addressed to JeongGil Ko; jgko@ajou.ac.kr
}

Received 16 June 2016; Accepted 12 July 2016

Academic Editor: Hyun-Ho Choi

Copyright ( $\odot 2016$ K. Lee and J. Ko. This is an open access article distributed under the Creative Commons Attribution License, which permits unrestricted use, distribution, and reproduction in any medium, provided the original work is properly cited.

As miniature-sized embedded computing platforms are ubiquitously deployed to our everyday environments, the issue of managing their power usage becomes important, especially when they are used in energy harvesting based self-organizing networks. One way to provide these devices with continuous power is to utilize RF-based energy transfer. Previous research in RF-based information and energy transfer builds up on the assumption that perfect channel estimation is easily achievable. However, as our preliminary experiments and many previous literature in wireless network systems show, making perfect estimations of the wireless channel is extremely challenging due to their quality fluctuations. To better reflect reality, in this work, we introduce an adaptive power allocation and splitting (APAS) scheme which takes imperfect channel estimations into consideration. Our evaluation results show that the proposed APAS scheme achieves near-optimal performances for transferring energy and data over a single RF transmission.

\section{Introduction}

As we slowly enter the era of the Internet of Things (IoT), we will start to experience various embedded computing systems being introduced to our everyday lives. In particular, it is important to maintain a long sensing and operational lifetime in self-organizing networks (SONs). Given that SONs are typically meant to tackle applications with little or no human intervention, their operational durations can determine the overall system's self-conguration, self-optimization, and selfhealing performance $[1,2]$. For this, a decade of research in the wireless embedded systems domain has introduced a number of schemes for optimizing energy efficiency on resource limited computing platforms [3-5].

In addition to these schemes that focus on conserving the power usage, another direction of research is to gather energy. This energy gathering can take two different forms where, in the first, an explicit hardware module is attached for harvesting energy from external sources (e.g., sunlight, wind, and vibration) $[6,7]$ and, in the second, the power generated from radio frequency (RF) signals can be used to transfer energy [8-10]. Given that the latter is only minimally affected by external environmental factors, we believe that it is an interesting research direction to explore. Since they do not require a large-sized energy harvesting unit, applying energy transfer techniques to data communications can effectively reduce the size of the the hardware used in lowpower wireless networking. Based on these benefits, in this work we study the possibilities of information and energy transfer using RF signals for powering low-power embedded computing platforms.

Given its attractiveness, a number of previous works have tried tackling interesting issues in various aspects of this research field. For example, in [11, 12], the authors investigated the theoretical performance limits for simultaneous wireless information and power transfer (SWIPT). The works in [13, 14] proposed time switching and dynamic power splitting for enabling efficient SWIPT. Furthermore, Nasir et al. took these findings to a networking perspective and introduced the relaying protocol in [15]. Here, the authors proposed a network where an energy constrained relay node harvests energy from the RF signals of a source node and uses this 
harvested energy for relaying information to the next hop. In [16], Shen et al. proposed transmitter designs for sumrate maximization with energy harvesting constraints on a multiple-input single-output (MISO) interference channel. In addition, an energy efficient resource allocation algorithm for SWIPT was investigated [17] and multiuser scheduling schemes for improving user fairness were studied in wireless networks with energy harvesting [18].

Despite these efforts from the research community, in this work we identify one important assumption that most of these previous works made. Namely, these works commonly took the assumption that wireless channels would be continuously stable and the communication quality would be perfect in all cases. However, research from the wireless networking systems community showed that this observation is far from being true. These RF signals can be severely impacted by external factors such as human movement, environmental changes, and even the time of day. Therefore, we believe that taking such real-world channel factors into consideration as we model the information and energy transfer behaviors of RF signals is important. Specifically, in this work we try to understand the performance of wireless links in reality and present a novel information and energy transfer model that reflects the nonperfect nature and inevitably imperfect channel quality estimations of real-world wireless environments. In addition, we propose an adaptive power allocation and splitting (APAS) scheme with considerations for imperfect wireless channel estimations in energy harvesting based SONs. Our evaluations show that APAS outperforms preexisting schemes and performs close to the optimal.

We summarize the contributions of this work threefold.

(i) We present empirical results on the RF characteristics in indoor environments to showcase the dynamics of real-world wireless channels. Our findings lead to a conclusion that perfect channel estimations are difficult to achieve due to various unexpected external factors.

(ii) We introduce a novel signal transfer model with channel estimation errors in consideration for analyzing the simultaneous transfer of information and energy using RF signals. Our model reflects realistic channel environments and, therefore, provides accurate performance bounds.

(iii) Using convex optimization techniques, we propose a resource allocation strategy that finds suboptimal transmission power allocation and power splitting ratios iteratively for a given training interval. Through simulations, we show that the proposed scheme provides near-optimal performance as well as considerable performance improvement compared to conventional schemes.

The rest of this paper is structured as follows. We start off the paper with an empirical study of real-world channel environments in Section 2. The findings from our preliminary studies become the basis of our system model presented in Section 3 and the proposed adaptive power allocation and splitting (APAS) scheme in Section 4. Using Section 5,

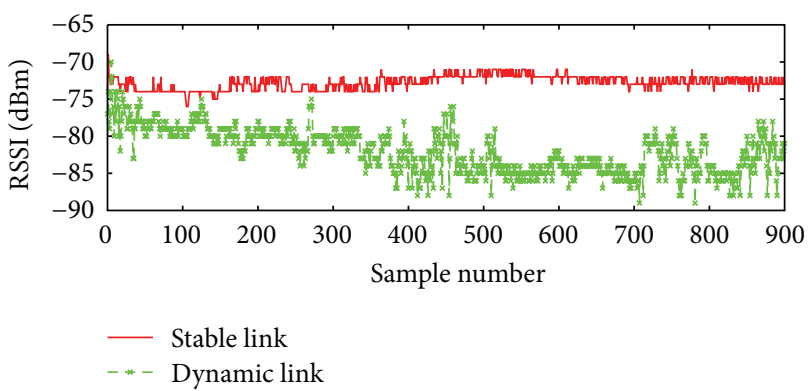

FIGURE 1: Empirical RSSI traces for stable and dynamic indoor environments.

we evaluate the performance of our proposed scheme and summarize our work in Section 6.

\section{Real Channel Environments}

Before we explain the details of our proposed scheme, we start by presenting empirical study results that show how dynamic a practical wireless channel environment can be. Specifically, we configure a transmitter node and a receiver node to be positioned $\sim 5$ meters apart. The transmitter node sends periodic packets with a packet transmission interval of $250 \mathrm{msec}$ at $0 \mathrm{dBm}$ and we test the wireless link in two different scenarios: one with no surrounding human movement activities (e.g., night-time) and another with continuous movement (e.g., day-time) between the two nodes. Since nodes were installed in a hallway environment with consistent human activities, the dynamic links in Figure 1 are sure to have high channel quality variability. In this experimental setting, we present the received signal strength indicator (RSSI) value observed at the receiver for incoming packets. Specifically, in Figure 1 we plot the RSSI over time for both the stable and dynamic links. Notice from Figure 1 that, with natural human-generated link dynamics (e.g., typical human movement behaviors), the signal strengths of incoming packets severely fluctuate.

These empirical results, though tested for a single environment, provide experimental evidence on the dynamics of real wireless channels. Using Figure 1, we try to show that schemes designed for real-world should not assume a stable wireless channel. This leads to an observation that perfect channel estimation can be difficult to achieve; thus, channel estimation analysis for information and energy transfer cannot be perfect in most cases. The remainder of this work builds up on such empirical findings to propose a model for analyzing the RF-based information and energy transfer under imperfect channel estimations.

\section{System Model}

Based on our observations of real-world channel environments, we take into consideration imperfect channel estimations in an orthogonal frequency division multiplexing (OFDM) based wireless point-to-point link consisting of a single transmitter $(\mathrm{Tx})$ and receiver $(\mathrm{Rx})$ pair, as shown in 
Figure 2. Tx and $\mathrm{Rx}$ nodes each are equipped with a singleantenna, and the frequency band is divided into independent $N$ subchannels. We assume that the subchannels follow a discrete time block-fading model in which the channel state is invariant for a transmission block interval $T$ [19, 20]. In addition, each real subchannel, $h_{n}$, is assumed to be an independent identically distributed complex random variable, such as $h_{n} \sim \mathscr{C} \mathscr{N}\left(0, \sigma_{h}^{2}\right)$ for $1 \leq n \leq N$. In a practical system, the channel estimation process obtains and exploits the channel state information (CSI). The transmission block $T$ is divided into two periods, where, in the first, we define $T_{\tau}$ for channel training and, in the second, we define the duration $T-T_{\tau}$ for data transmission. The minimum mean square error (MMSE) criterion is assumed to be used for estimating the channel status, and each estimated subchannel, $\widehat{h}_{n}$, follows a Gaussian distribution, such as $\widehat{h}_{n} \sim \mathscr{C} \mathcal{N}\left(0, \sigma_{\widehat{h}}^{2}\right)$ for $1 \leq n \leq N$.

Under such settings, we note that the RF signals (at the $\mathrm{Rx}$ node) can be used in two ways, either for information decoding (ID) or for energy harvesting (EH), while these modes cannot take place simultaneously. Here, the received signal is split in two portions in each subchannel; the portion of $\rho_{n}$ among $T-T_{\tau}$ is reserved for ID, while $1-\rho_{n}$ is for $\mathrm{EH}$ before performing active analog or digital signal processing. (We assume that the $\mathrm{Rx}$ node is equipped with a perfect passive power splitting unit [17].) In addition, as RF signals are split in two streams, two types of noise should be considered: antenna noise, $n_{A}$, and signal processing noise, $n_{S} \cdot n_{A}$ is generated at the $\mathrm{Rx}$ antenna while $n_{S}$ is introduced when the received signal is divided into ID and $\mathrm{EH}$. Nevertheless, we neglect $n_{A}$ since it is much smaller than $n_{S}$ [14]. Furthermore, we assume $n_{S} \sim \mathscr{C} \mathscr{N}(0,1)$. Then, the achievable sum rate using the estimated subchannels can be represented by [20]

$$
\begin{aligned}
R & =\sum_{n=1}^{N} r_{n} \\
& =\sum_{n=1}^{N} \frac{T-T_{\tau}}{T} \log _{2}\left(1+\frac{\left(1+p_{\tau} T_{\tau}\right) \rho_{n} p_{n}\left|\widehat{h}_{n}\right|^{2}}{1+\rho_{n} p_{n}+p_{\tau} T_{\tau}}\right),
\end{aligned}
$$

where $p_{\tau}$ and $p_{n}$ denote the power for estimating channel conditions and power allocated in subchannel $n$ for data transmission, respectively. When performing $\mathrm{EH}$ at the $\mathrm{Rx}$, the harvested energy is represented as

$$
\sum_{n=1}^{N} g_{n}=\sum_{n=1}^{N} \frac{T-T_{\tau}}{T} \eta\left|\widehat{h}_{n}\right|^{2}\left(1-\rho_{n}\right) p_{n}
$$

where $\eta$ is the energy conversion efficiency achieved by converting the received RF signals into harvestable energy at the $\mathrm{Rx}$. We also assume that $\sum_{n=1}^{N} \eta\left|h_{n}\right|^{2} \leq 1$, according to the laws of thermodynamics.

We now look into the training interval, the power allocation, and the power splitting ratio required to maximize the sum rate while guaranteeing the minimum harvested energy $g_{\text {min }}$. Consider

$$
\begin{array}{ll}
\max _{T_{\tau}, \vec{p}, \vec{\rho}} & \sum_{n=1}^{N} r_{n} \\
\text { s.t. } \quad \text { C1: } \sum_{n=1}^{N} g_{n} \geq g_{\min }, \\
\text { C2: } \sum_{n=1}^{N} p_{n} \leq p_{\max }, \\
\text { C } 3: p_{n} \geq 0, \quad \forall n, \\
\text { C4: } 0 \leq \rho_{n} \leq 1, \quad \forall n, \\
\text { C5: } 0 \leq T_{\tau} \leq T .
\end{array}
$$

Here, $\vec{p}=\left\{p_{1}, \ldots, p_{N}\right\}$ and $\vec{\rho}=\left\{\rho_{1}, \ldots, \rho_{N}\right\}$. Furthermore, the five constraints can be explained as follows. Constraint $\mathrm{C} 1$ ensures that the amount of energy harvested should be larger than the minimum amount of required energy $g_{\min }$. Constraint C2 limits the available transmission power of the Tx to $p_{\max }$. Constraint $\mathrm{C} 3$ is a nonnegative constraint on the transmission power. Constraints $\mathrm{C} 4$ and C5 are the ranges of $\rho_{n}$ and $T_{\tau}$, respectively.

\section{Adaptive Power Allocation and Splitting}

We now propose an adaptive power allocation and splitting (APAS) algorithm by solving the optimization problem in (3). We note that it is difficult to find a closed form solution for $T_{\tau}^{*}$ from optimization techniques, given that the objective function of (3) is not in concave form with respect to $T_{\tau}$. However, considering the fact that $T_{\tau}$ lies within the interval $(0, T), T_{\tau}^{*}$ can be derived using a one-dimensional exhaustive search. For example, $T_{\tau}^{*}$ can be found from the probability density function (PDF) of channel distribution. If $T_{\tau}^{*}$ is determined at once, it can be used for estimating all unknown channels that will be generated. Furthermore, using the concavity of $\vec{p}$ and $\vec{\rho}$, we can find their suboptimal values iteratively, which reflect channel estimation errors. (In a biconvex problem, where the problem in (3) is convex with respect to $\vec{p}$ for a fixed $\vec{\rho}$ or vice versa, the convergence of a partial optimum solution can be guaranteed by using the block coordinate descent (BCD) algorithm [21].)

For a given $T_{\tau}$, Tx estimates CSI on subchannels and determines the allocated power and the power splitting ratio. The Lagrangian function of (3) can be expressed by

$$
\begin{aligned}
\Lambda(\vec{p}, \vec{\rho}, \lambda, \mu)= & \sum_{n=1}^{N} r_{n}+\lambda\left(\sum_{n=1}^{N} g_{n}-g_{\min }\right) \\
& +\mu\left(p_{\max }-\sum_{n=1}^{N} p_{n}\right) .
\end{aligned}
$$

Here, $\lambda$ and $\mu$ are nonnegative Lagrangian multipliers, which correspond to constraints $\mathrm{C} 1$ and $\mathrm{C} 2$, respectively. To find a solution to this, we decouple the original problem into parallel $N$ subproblems for each subchannel. By discarding the 


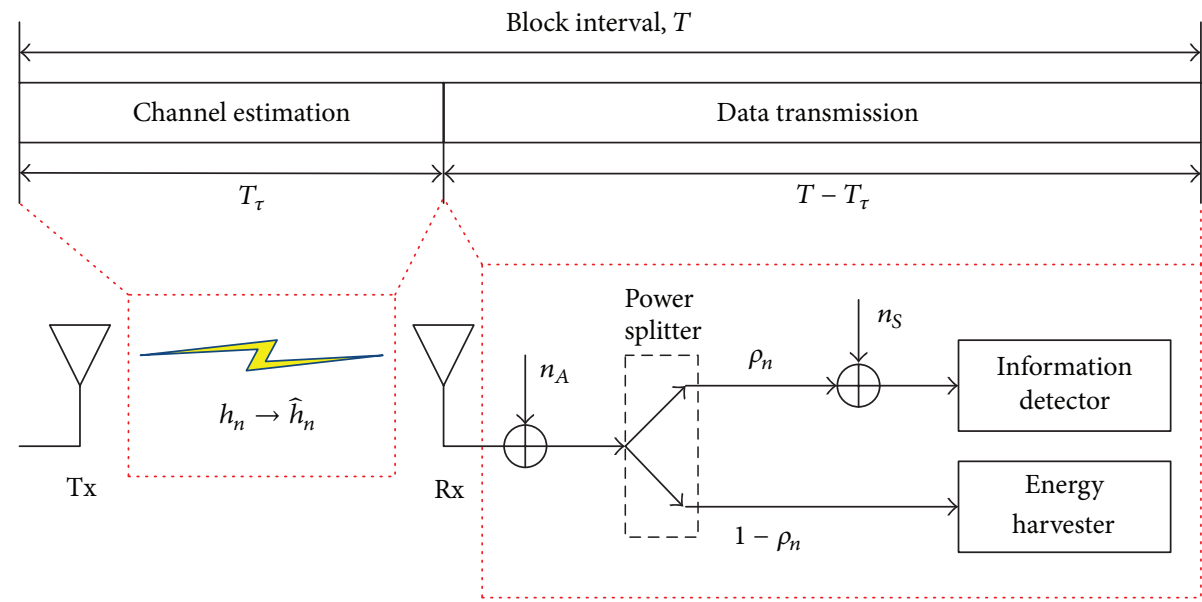

FIGURE 2: System model for wireless information and energy transfer.

constant terms, the Lagrangian function (4) for a particular subchannel $n$ can be represented as

$$
\Lambda\left(p_{n}, \rho_{n}, \lambda, \mu\right)=r_{n}+\lambda g_{n}-\mu p_{n} .
$$

By taking the derivative of (5) with respect to $p_{n}$, we can obtain a power allocation strategy as in the following:

$$
\begin{aligned}
p_{n}^{*} & =\left[\frac{H_{e}}{1+H_{e}\left|\widehat{h}_{n}\right|^{2}}\right. \\
& \cdot \sqrt{\frac{4 \rho_{n}\left|\widehat{h}_{n}\right|^{2}\left(1+H_{e}\left|\widehat{h}_{n}\right|^{2}\right)+(\ln 2) H_{e}^{2}\left|\widehat{h}_{n}\right|^{4} \zeta_{n}}{4(\ln 2) \rho_{n}^{2} \zeta_{n}}} \\
& \left.-\frac{H_{e}\left(2+H_{e}\left|\widehat{h}_{n}\right|^{2}\right)}{2 \rho_{n}\left(1+H_{e}\left|\widehat{h}_{n}\right|^{2}\right)}\right]^{+} .
\end{aligned}
$$

Here, $[x]^{+}=\max (0, x), H_{e}=1+p_{\tau} T_{\tau}$, and $\zeta_{n}=(T /(T-$ $\left.\left.T_{\tau}\right)\right) \mu-\lambda \eta\left|\widehat{h}_{n}\right|^{2}\left(1-\rho_{n}\right)$. For the obtained $p_{n}^{*}$ from (6), we also take the derivative of (5) with respect to $\rho_{n}$, to obtain the power splitting strategy as in the following:

$$
\begin{aligned}
\rho_{n}^{*} & =\left[\frac{H_{e}}{1+H_{e}\left|\widehat{h}_{n}\right|^{2}}\right. \\
& \cdot \sqrt{\frac{4\left|\widehat{h}_{n}\right|^{2}\left(1+H_{e}\left|\widehat{h}_{n}\right|^{2}\right)+(\ln 2) H_{e}^{2} \lambda \eta\left|\widehat{h}_{n}\right|^{6}}{4(\ln 2) p_{n}^{2} \lambda \eta\left|\widehat{h}_{n}\right|^{2}}} \\
& \left.-\frac{H_{e}\left(2+H_{e}\left|\widehat{h}_{n}\right|^{2}\right)}{2 p_{n}\left(1+H_{e}\left|\widehat{h}_{n}\right|^{2}\right)}\right]_{0}^{1} .
\end{aligned}
$$

Here, $[x]_{0}^{1}=\min (\max (0, x), 1)$.
Then, $p_{n}^{*}$ and $\rho_{n}^{*}$ can be interpreted in terms of channel estimation, channel condition, and harvested energy as follows. We especially note that an increment in $T_{\tau}$ ensures the exact estimation of channel conditions but decreases the data transmission time. An extremely large $T_{\tau}$ does not allow for a dedicated data transmission time; as a result, $p_{n}^{*}$ and $\rho_{n}^{*}$ become zero. In addition, $p_{n}^{*}$ and $\rho_{n}^{*}$ are proportional to $\left|\widehat{h}_{n}\right|^{2}$, so they show large values on subchannels with good channel conditions. $p_{n}^{*}$ is proportional to $\lambda \eta\left|\widehat{h}_{n}\right|^{2}$, which is related to the amount of harvested energy, but $\rho_{n}^{*}$ is inversely proportional to $\lambda \eta\left|\widehat{h}_{n}\right|^{2}$. An amount of $p_{n}^{*}$ increases on a good subchannel, where large amounts of energy harvesting are possible, while $\rho_{n}^{*}$ decreases on that subchannel to meet the constraint $\mathrm{Cl}$ for harvested energy tightly. In short, $p_{n}^{*}$ and $\rho_{n}^{*}$ are adjusted reciprocally to the maximize sum rate while guaranteeing $g_{\text {min }}$.

Based on the obtained $\overrightarrow{p^{*}}$ and $\overrightarrow{\rho^{*}}$, Lagrangian multipliers can be updated by a well-known bisection algorithm or a gradient algorithm. We detail the overall procedure of the proposed algorithm in Algorithm 1.

\section{Simulation Results and Discussion}

For evaluations, we configure a simulation environment as in the following. Specifically, we set $N=32, T=1000$, $\eta=0.9, p_{\tau}=p_{\max }=43 \mathrm{dBm}$, and $g_{\min }=20 \mathrm{dBm}$. We assume that subchannel $h_{n}$ experiences Rayleigh fading, so $h_{n}$ is generated as a random variable distributed exponentially with $\sigma_{h}^{2}=10^{-4}$. In addition, it is independent from other subchannels $h_{j}$ for $j \neq n$. We compare the performance of APAS with three algorithms: OPAS, EPAS, and $E^{2}$ PAS.

(i) Optimal power allocation and splitting (OPAS): with perfect CSI at the Tx (CSIT), $\vec{p}$ and $\vec{\rho}$ are determined optimally.

(ii) Equal Power Allocation and Adaptive Power Splitting (EPAS): power is allocated equally to all subchannels, and $\vec{\rho}$ is determined adaptively to meet $g_{\min }$ with respect to (7). 

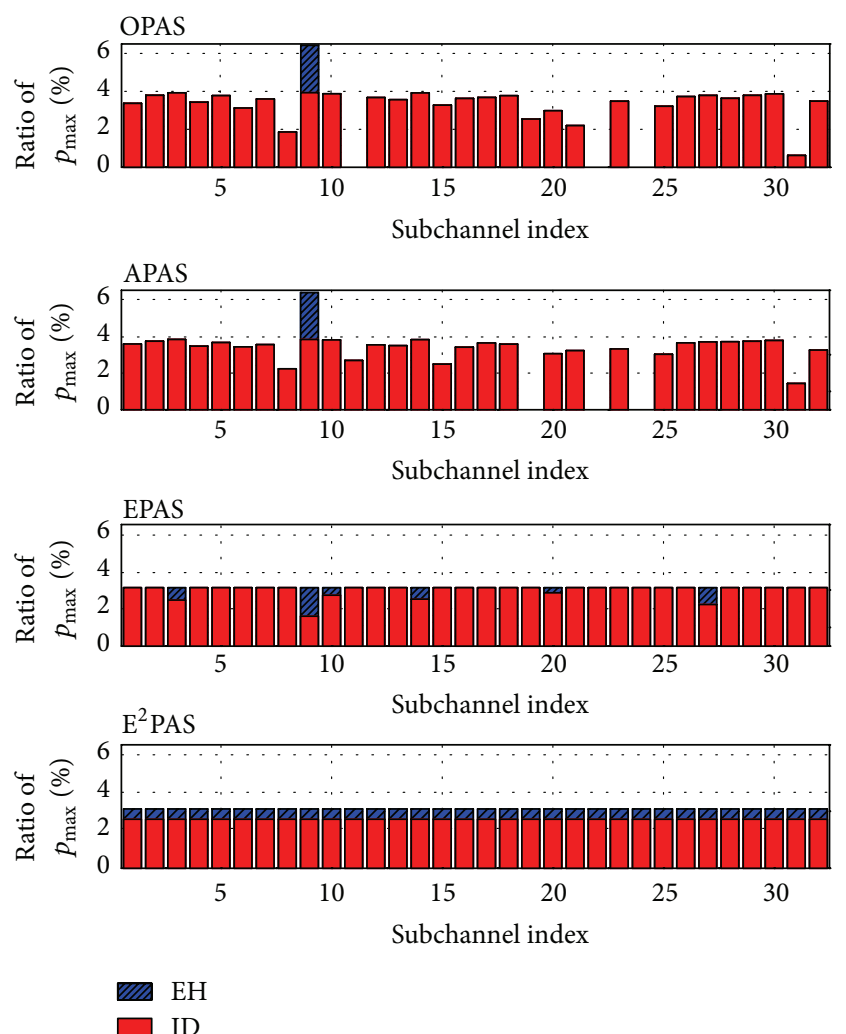

Figure 3: Power allocation and splitting of OPAS, APAS, EPAS, and $\mathrm{E}^{2} \mathrm{PAS}$.

(1) Initialize $\vec{p}, \vec{\rho}$, and Lagrangian multipliers

(2) for $T_{\tau}=1: T$

(3) Estimate $\left|\widehat{h}_{n}\right|^{2}$ for $\forall n$ based on the PDF of channel

(4) Evaluate $R$

(5) end for

(6) $\operatorname{Return} T_{\tau}^{*}=\max _{T_{\tau}} R$

(7) repeat

(8) Find $\overrightarrow{p^{*}}$ according to (6)

(9) Find $\overrightarrow{\rho^{*}}$ according to (7)

(10) Update Lagrangian multipliers, $\lambda$ and $\mu$

(11) until $\overrightarrow{p^{*}}$ and $\overrightarrow{\rho^{*}}$ converge

Algorithm 1: Adaptive power allocation and splitting.

(iii) Equal Power Allocation and Equal Power Splitting ( $\left.\mathrm{E}^{2} \mathrm{PAS}\right)$ : power is allocated equally to all subchannels, and $\vec{\rho}$ is determined equally for all subchannels to meet $g_{\text {min }}$.

Figure 3 shows the power allocation and splitting of OPAS, APAS, EPAS, and $\mathrm{E}^{2} \mathrm{PAS}$, respectively. In OPAS and APAS, a large amount of power is allocated to the subchannel with the highest channel gain, and a portion of power is split to harvest energy on that subchannel. It is best to use the power allocated to the best subchannel for guaranteeing $g_{\text {min }}$ since energy can be harvested with higher efficiency.

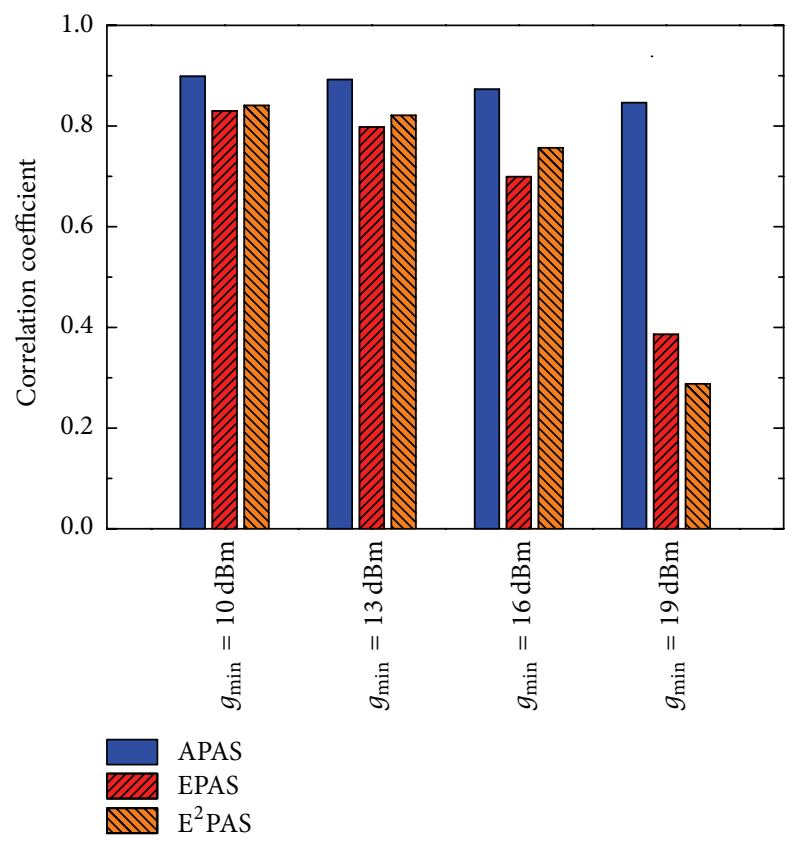

Figure 4: Correlation coefficient of APAS, EPAS, and $\mathrm{E}^{2}$ PAS.

The differences of resource allocation between OPAS and APAS come from the errors in channel estimation, but their resource allocation forms show similar tendency. This indicates that APAS can achieve a performance close to the optimal bound. On the other hand, in EPAS and $\mathrm{E}^{2} \mathrm{PAS}$, power is allocated equally on all subchannels and energy is harvested on several subchannels; therefore, a subset of the power can be used inefficiently. In particular, despite its implementation simplicity, performance can be degraded severely in $E^{2}$ PAS due to the fact that resource allocation is performed regardless of the channel conditions.

Figure 4 shows the correlation coefficient of APAS, EPAS, and $\mathrm{E}^{2} \mathrm{PAS}$ with varying $g_{\min }$. This result targets for showing how the power allocation and splitting of each scheme is similar to OPAS. In OPAS, more power is allocated to the best subchannel to ensure $g_{\text {min }}$ with a high efficiency as $g_{\text {min }}$ increases. As a result, we can notice here that the correlation coefficients of EPAS and $\mathrm{E}^{2} \mathrm{PAS}$ decrease seriously. On the other hand, the correlation coefficient of APAS stays high, $\sim 0.9$, even with increasing $g_{\text {min }}$. Therefore, this result suggests that APAS can adapt the power allocation and splitting strategy to a level similar to the optimal solution under various conditions.

Figure 5 plots the data rate $R$ versus the training interval $T_{\tau}$, which shows the effects of $T_{\tau}$ on $R$. As $T_{\tau}$ increases, it is possible to estimate the channel conditions more accurately. As a result, $R$ increases gradually to a peak point. However, additional increase in $T_{\tau}$ beyond its optimal value causes reduction in the dedicated transmission time, thereby decreasing $R$. This suggests that there is an optimal value of $T_{\tau}$ for maximizing $R$ from the tradeoff relationship between the accuracy of channel estimations and the duration of data transmission time. The optimal training interval $T_{\tau}^{*}$ increases with a large $g_{\min }$, which indicates that an exact channel 


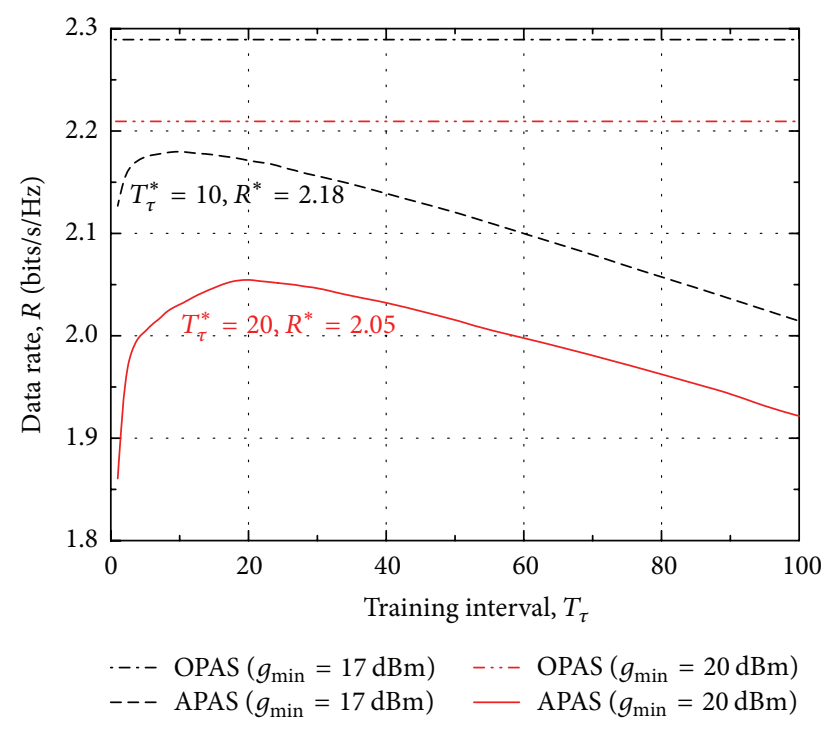

Figure 5: Data rate $R$ versus training interval $T_{\tau}$.

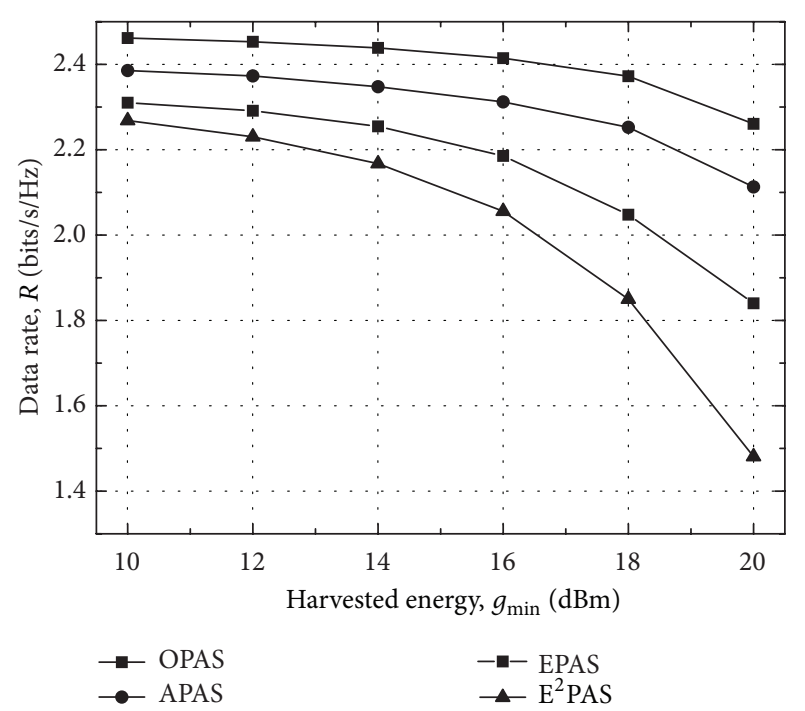

FIgURE 6: Data rate $R$ versus harvested energy $g_{\min }$ when $T_{\tau}=20$.

estimation is desired to satisfy a large requirement for the harvested energy. There is no significant difference, in particular less than $10 \%$, between the maximum $R$ achieved at $T_{\tau}^{*}$ and the optimal bound of $R$.

Figure 6 shows the data rate $R$ versus the harvested energy $g_{\min }$ when $T_{\tau}=20$. As $g_{\min }$ increases, a large portion of power should be used for harvesting energy. In consequence, $R$ decreases gradually for all algorithms. APAS has the gain of adaptive power allocation compared with EPAS, while it has the gain of adaptive power allocation and splitting when compared to $E^{2}$ PAS. Therefore, we can confirm the gain of each adaptive strategy by comparing APAS with EPAS and $\mathrm{E}^{2} \mathrm{PAS}$, respectively. In EPAS and $\mathrm{E}^{2} \mathrm{PAS}$, the rates at which $R$ decreases with increasing $g_{\min }$ are noticeably steeper than those of OPAS and APAS. This is mainly due to the fact that, in EPAS and $\mathrm{E}^{2} \mathrm{PAS}$, the power cannot be used efficiently.
Therefore, APAS outperforms EPAS and $E^{2}$ PAS significantly at larger $g_{\min }$ values. For example, APAS outperforms EPAS and $\mathrm{E}^{2} \mathrm{PAS}$ in terms of $R$ by $20 \%$ and $40 \%$ at $g_{\min }=20 \mathrm{dBm}$, respectively. On the other hand, the performance difference between OPAS and APAS remains relatively constant despite increasing $g_{\text {min }}$.

\section{Conclusion}

RF-based information and energy transferring techniques hold the potential to dramatically change the design of wireless systems and their networking architectures. Nevertheless, the research community is still in the early stages of validating their effectiveness. In this work, we target to tackle one of the strongest assumptions that most of the works in information and energy transfer made, which is the assumption that perfect channel estimation is possible. We show through an empirical study that the variability of wireless channels makes perfect estimations of the wireless environment close to impossible. For this, we propose APAS, which takes into consideration imperfect channel estimation results for evaluating the effectiveness of information and energy transfer on wireless devices for energy harvesting based SONs. In APAS, the power allocation and splitting ratio is determined adaptively with considerations for the estimated channel quality. In addition, our results indicate that APAS achieves near-optimal performances under various conditions. We hope that this work can act as a catalyst in enabling future research that tries to adopt RF-based information and energy transfer in realistic channel environments.

\section{Competing Interests}

The authors declare that there is no conflict of interests regarding the publication of this paper.

\section{Acknowledgments}

This research was supported by Basic Science Research Program through the National Research Foundation of Korea (NRF) funded by the Ministry of Science, ICT \& Future Planning (2015R1C1A1A01051747).

\section{References}

[1] H. Hu, J. Zhang, X. Zheng, Y. Yang, and P. Wu, "Selfconfiguration and self-optimization for LTE networks," IEEE Communications Magazine, vol. 48, no. 2, pp. 94-100, 2010.

[2] 3GPP, "Self-organizing networks (SON): concepts and requirements," 3GPP TS 32.500, 2008, V8.0.0.

[3] G. Y. Li, Z. Xu, C. Xiong et al., "Energy-efficient wireless communications: tutorial, survey, and open issues," IEEE Wireless Communications, vol. 18, no. 6, pp. 28-35, 2011.

[4] C. Han, T. Harrold, S. Armour et al., "Green radio: radio techniques to enable energy-efficient wireless networks," IEEE Communications Magazine, vol. 49, no. 6, pp. 46-54, 2011.

[5] H. Bogucka and A. Conti, "Degrees of freedom for energy savings in practical adaptive wireless systems," IEEE Communications Magazine, vol. 49, no. 6, pp. 38-45, 2011.

[6] H. Kulah and K. Najafi, "Energy scavenging from low-frequency vibrations by using frequency up-conversion for wireless sensor 
applications," IEEE Sensors Journal, vol. 8, no. 3, pp. 261-268, 2008.

[7] S. Sudevalayam and P. Kulkarni, "Energy harvesting sensor nodes: survey and implications," IEEE Communications Surveys and Tutorials, vol. 13, no. 3, pp. 443-461, 2011.

[8] T. Le, K. Mayaram, and T. Fiez, "Efficient far-field radio frequency energy harvesting for passively powered sensor networks," IEEE Journal of Solid-State Circuits, vol. 43, no. 5, pp. 1287-1302, 2008.

[9] R. J. M. Vullers, R. van Schaijk, I. Doms, C. Van Hoof, and R. Mertens, "Micropower energy harvesting," Solid-State Electronics, vol. 53, no. 7, pp. 684-693, 2009.

[10] M. Piñuela, P. D. Mitcheson, and S. Lucyszyn, "Ambient RF energy harvesting in urban and semi-urban environments," IEEE Transactions on Microwave Theory and Techniques, vol. 61, no. 7, pp. 2715-2726, 2013.

[11] L. R. Varshney, "Transporting information and energy simultaneously," in Proceedings of the IEEE International Symposium on Information Theory (ISIT '08), pp. 1612-1616, IEEE, Toronto, Canada, July 2008.

[12] P. Grover and A. Sahai, "Shannon meets tesla: wireless information and power transfer," in Proceedings of the IEEE International Symposium on Information Theory (ISIT '10), pp. 2363-2367, IEEE, Austin, Tex, USA, June 2010.

[13] L. Liu, R. Zhang, and K.-C. Chua, "Wireless information transfer with opportunistic energy harvesting," IEEE Transactions on Wireless Communications, vol. 12, no. 1, pp. 288-300, 2013.

[14] L. Liu, R. Zhang, and K.-C. Chua, "Wireless information and power transfer: a dynamic power splitting approach," IEEE Transactions on Communications, vol. 61, no. 9, pp. 3990-4001, 2013.

[15] A. A. Nasir, X. Zhou, S. Durrani, and R. A. Kennedy, "Relaying protocols for wireless energy harvesting and information processing," IEEE Transactions on Wireless Communications, vol.12, no. 7, pp. 3622-3636, 2013.

[16] C. Shen, W.-C. Li, and T.-H. Chang, "Wireless information and energy transfer in multi-antenna interference channel," IEEE Transactions on Signal Processing, vol. 62, no. 23, pp. 6249-6264, 2014.

[17] D. W. K. Ng, E. S. Lo, and R. Schober, "Wireless information and power transfer: energy efficiency optimization in OFDMA systems," IEEE Transactions on Wireless Communications, vol. 12, no. 12, pp. 6352-6370, 2013.

[18] R. Morsi, D. S. Michalopoulos, and R. Schober, "Multiuser scheduling schemes for simultaneous wireless information and power transfer over fading channels," IEEE Transactions on Wireless Communications, vol. 14, no. 4, pp. 1967-1982, 2015.

[19] R. A. Berry and R. G. Gallager, "Communication over fading channels with delay constraints," IEEE Transactions on Information Theory, vol. 48, no. 5, pp. 1135-1149, 2002.

[20] B. Hassibi and B. M. Hochwald, "How much training is needed in multiple-antenna wireless links?" IEEE Transactions on Information Theory, vol. 49, no. 4, pp. 951-963, 2003.

[21] J. Gorski, F. Pfeuffer, and K. Klamroth, "Biconvex sets and optimization with biconvex functions: a survey and extensions," Mathematical Methods of Operations Research, vol. 66, no. 3, pp. 373-407, 2007. 

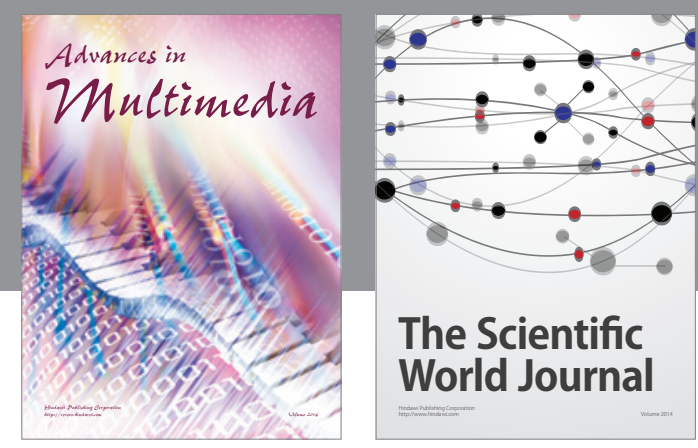

The Scientific World Journal
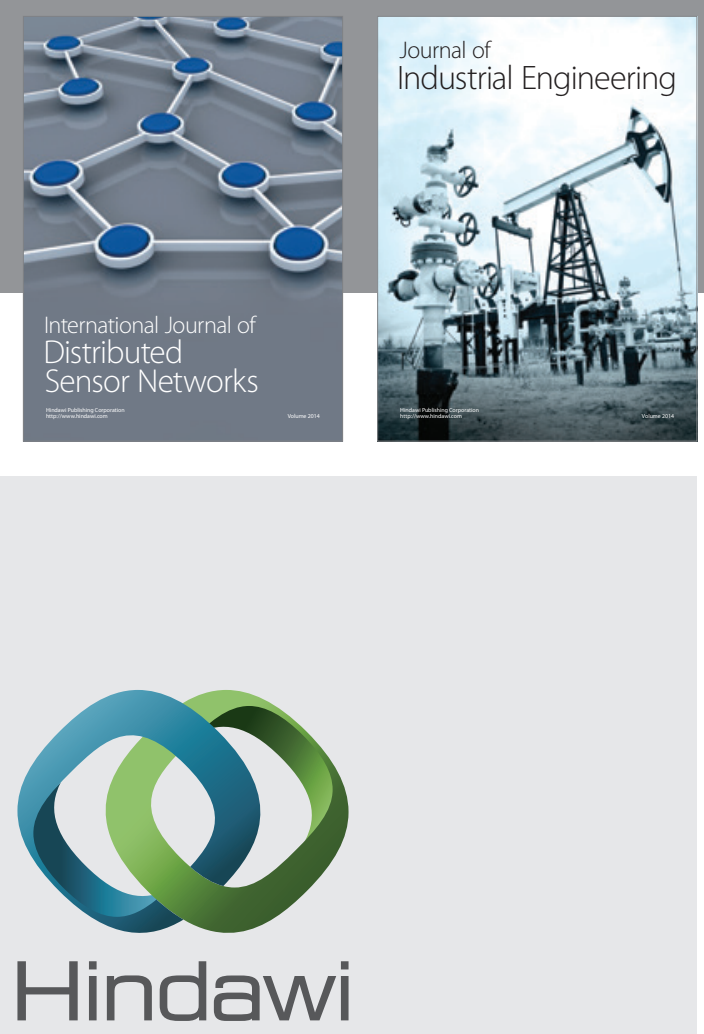

Submit your manuscripts at

http://www.hindawi.com

\section{Computer Networks} and Communications
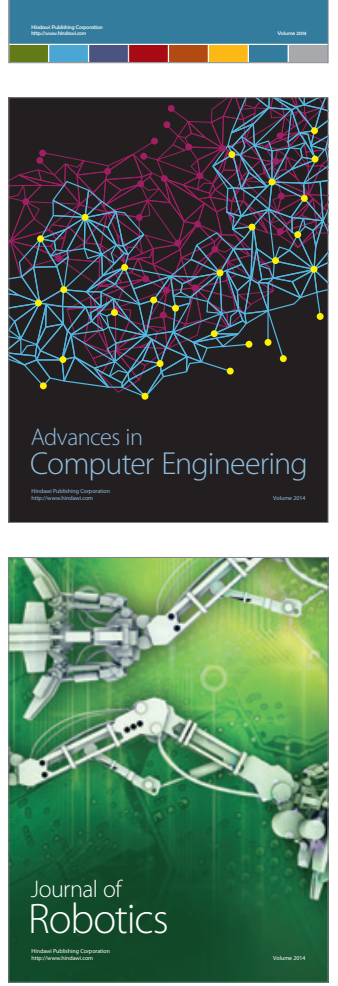
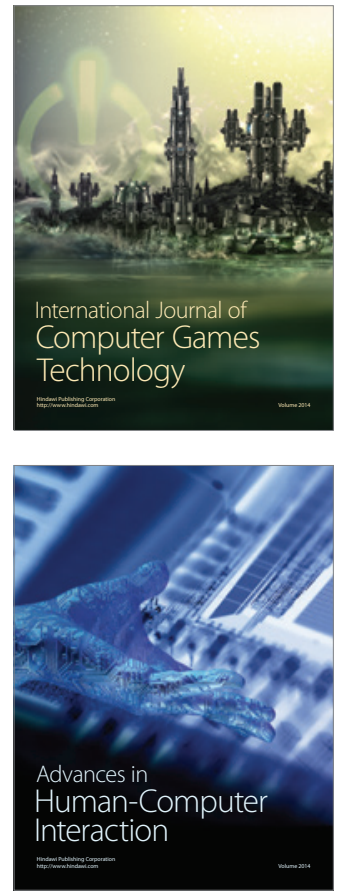
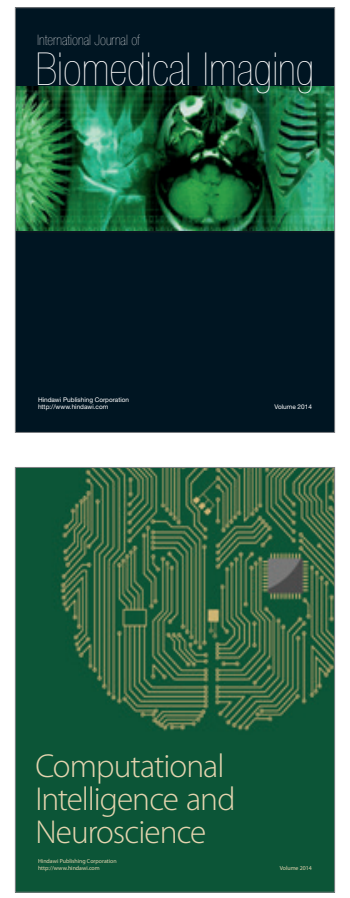
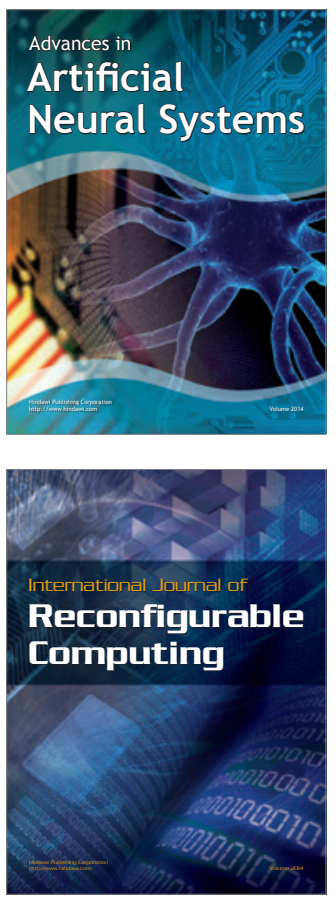
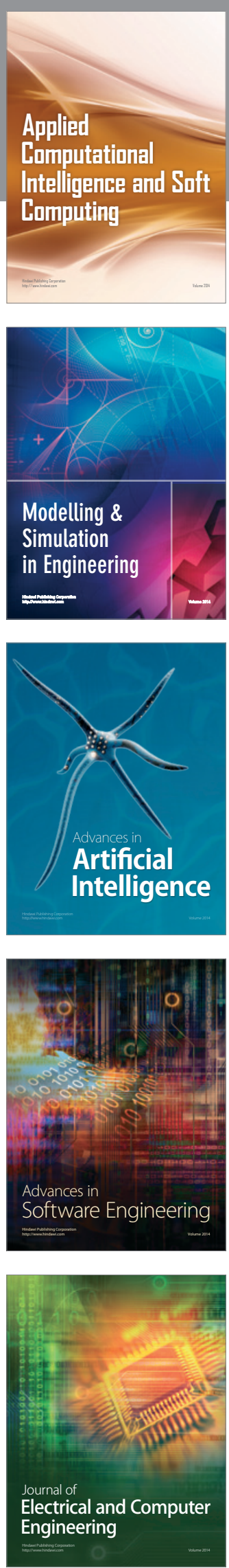